\title{
Implantation of the Subcutaneous Implantable Cardioverter-Defibrillator with Truncal Plane blocks: Expanding ERAS to Cardiology
}

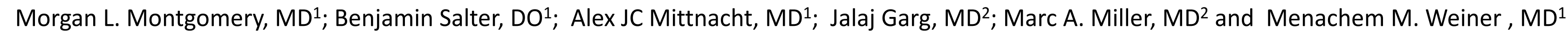
${ }^{1}$ Department of Anesthesiology and ${ }^{2}$ Helmsley Electrophysiology Center in the Department of Cardiology and the 2, Icahn School of Medicine at Mount Sinai, New York, New York

Background and Aims: The subcutaneous implantable cardioverter-defibrillator (S-ICD) is an attractive alternative to conventional transvenous ICDs Due to the nature of the procedure itself, the S-ICD is associated with a unique set of implant challenges and barriers to wider adoption. Some of these barriers include the frequent use of general anesthesia (GA) and significant post-operative pain. However, there are disadvantages in the routine use of GA for S-ICD implantation, including hemodynamic compromise, the typical risks related to endotracheal intubation, as well as patient discomfort due to nausea and vomiting frequently seen in patients undergoing GA. Post-operative analgesia usually consists of opioids; however, their use is associated with substantial short- and long-term morbidity. Accordingly, there is an increased interest in multimodal pain management strategies, including the use of regional anesthetic techniques, that adequately address peri/post-operative pain control with minimal narcotic use.

Methods: Single-center analysis of S-ICD implantation which compared: i) GA and local wound infiltration (GA + LAWI) ( $n=$ $10)$, ii) $G A$ with truncal plane blocks $(G A+T B L)(n=10)$ and iii) pre-operative analgesics (acetaminophen + gabapentin) with deep sedation and truncal plane blocks (DS +TBL) $(n=10)$. The adjunctive truncal plane blocks include serratus anterior fascial plane block and transversus thoracic plane block. Opioid consumption was calculated as milligram morphine equivalents (MME).

Results: In the first 12 hours, opioid consumption was significantly lower in the patients implanted with DS + TBL (MME $=0)$, as compared with patients receiving $G A+T B L(M M E=45 ; p$ $=0.003$ ) or GA + LAWI (MME $=75 ; p=0.003$ ) (Figure). Total inhospital opioid-consumption was also lowest in the DS + TBL cohort. There was a trend towards lower vasopressor administration (norepinephrine equivalents [NE]) in patients that were implanted with $\mathrm{DS}+\mathrm{TBL}(\mathrm{NE}=96 \mu \mathrm{g}$ ) compared to GA + LAWI (NE = $611 \mu \mathrm{g}, \mathrm{p}=0.08)$.

Conclusions: Subcutaneous ICD implantation with anesthesiadelivered deep sedation and a multimodal anesthetic regimen which includes truncal plane blocks is feasible and associated with significantly less peri-operative opioid-consumption. Future studies will be needed to confirm these encouraging results, and determine if routine use of this anesthetic and analgesic strategy facilitates improved pain control and shorter recovery times.
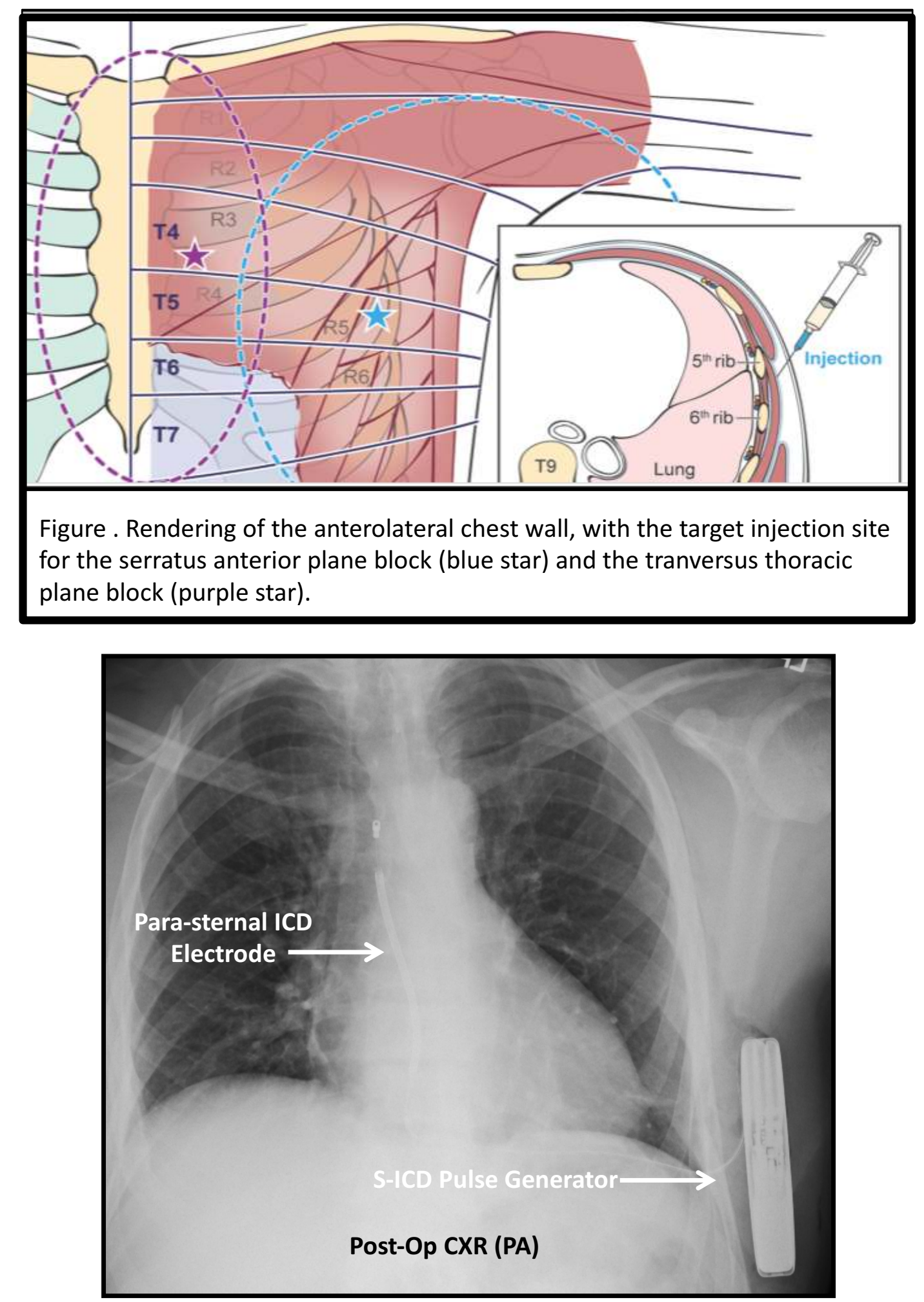

Figure. Anesthetic and analgesic protocols for Subcutaneous ICD implantation
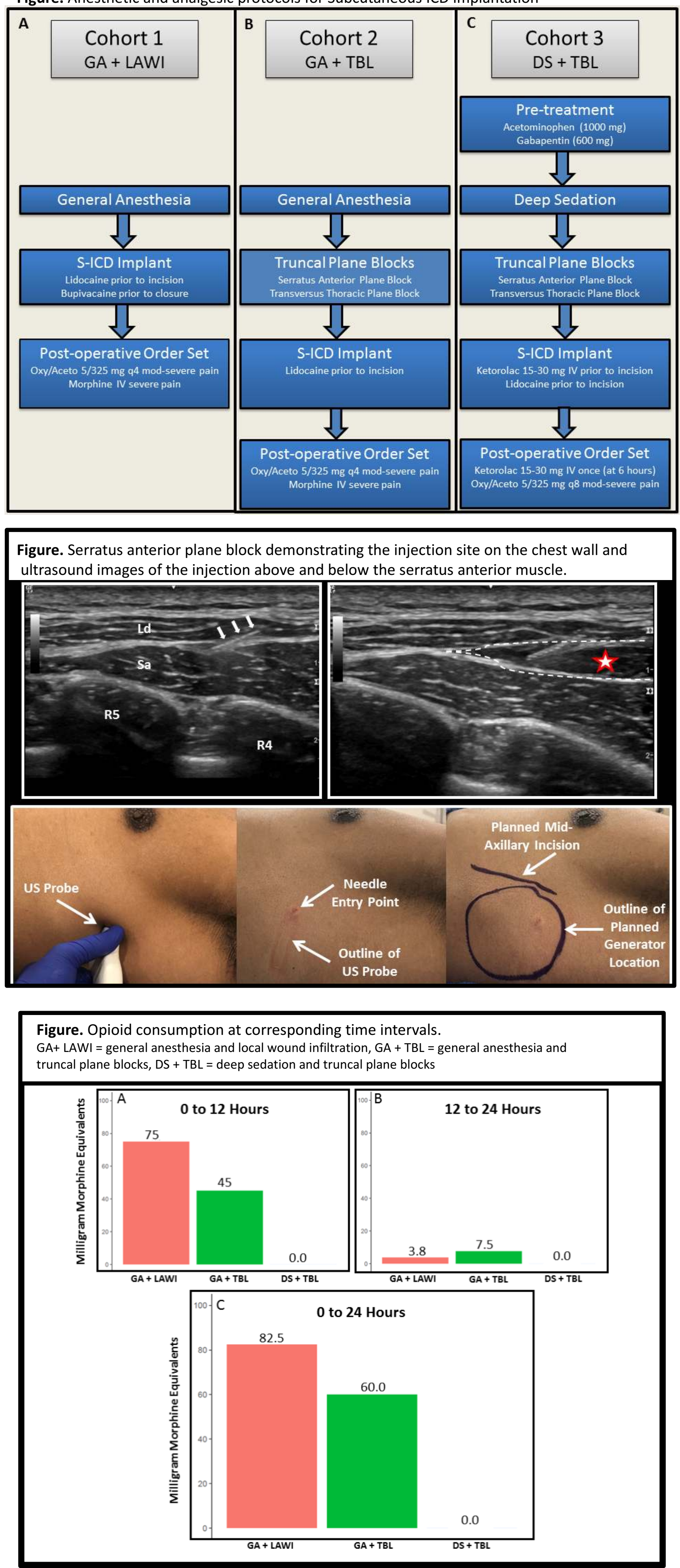